\title{
Absence of the set-size effect in memory-search tasks in the absence of a preprobe delay
}

\author{
DON DIENER \\ University of Nevada, Las Vegas, Nevada
}

\begin{abstract}
In the traditional Sternberg (1966) paradigm, response latency increases linearly with increases in the size of the positive set (the set-size effect). The results of four experiments converge on the conclusion that this set-size effect depends on a delay before the presentation of the memory probe. In Experiment 1, subjects were required to respond as soon as a repetition occurred in a series of digits. Despite the similarity of this task to memory-search tasks that invariably show set-size effects, there was no increase in response latency with increasing series length. Neither the inclusion of negative trials (Experiment 2) nor the explicit designation of the test digit (Experiment 3) resulted in the typical set-size effect. However, the introduction of a 1-sec preprobe delay (Experiment 4) resulted in a set-size effect of $31 \mathrm{msec} / \mathrm{item}$.
\end{abstract}

In a classic series of experiments, Sternberg (1966, $1969)$ presented subjects with a memory set consisting of a series of one to six different digits. This "positive set" was followed in about $2 \mathrm{sec}$ by a single "probe" digit. The subjects' task was to indicate, by depressing the appropriate lever, whether the probe was a member of the positive set. Important results of these experiments included a linear increase in response latency with an increase in the size of the positive set (about $38 \mathrm{msec} / \mathrm{item}$ ) and equal slopes of the functions relating response latency to set size for positive and negative responses. The set-size effect led Sternberg to conclude that the memory search required the serial comparison of the probe to members of the positive set. The equality of the slopes for positive and negative responses suggested that the search is exhaustive, that is, that the serial-comparison process does not end when a match is found but continues until the probe has been compared to every item in the set.

Sternberg's $(1966,1969)$ procedures have been widely replicated and elaborated. Members of the positive set have been drawn from such categories as letters, words, colors, and shapes (see Cavanagh, 1972, for a review). The rate of presentation of items in the positive set has been varied, as has the interval between the presentation of the positive set and the memory probe (see Cavanagh, 1976, for a discussion). The probe stimulus has been presented to a different sensory modality or in a different format from members of the positive set (Sternberg, 1975). Concurrent processing demands have been imposed on subjects during the memory search (e.g., Howard, 1975; Krueger, 1975). Linear set-size effects are invariably found under these and other experimental conditions, although the slope of the function relating

I wish to thank Kim Foster for her assistance in collecting the data Requests for reprints should be addressed to Don Diener, Department of Psychology, University of Nevada, Las Vegas, 4505 Maryland Parkway, Las Vegas, NV 89154 response latency to set size, its zero intercept, or both are often affected by experimental manipulations (see Sternberg, 1975, for a discussion).

Because it is found under such a wide variety of experimental conditions, the set-size effect has been regarded as a general characteristic of the retrieval of information from short-term memory, rather than a task-specific phenomenon. All seriously entertained models of shortterm memory retrieval formulated since the publication of Sternberg's (1966) results have been forced to deal with the effect. Indeed, many models were formulated specifically to account for Sternberg's findings. Because the idea of an exhaustive search is counterintuitive, and because most perceptual and cognitive phenomena appear to involve relatively slow parallel processes rather than highspeed serial processes, alternative models usually have assumed parallel rather than serial access to the contents of short-term memory (e.g., Baddeley \& Ecob, 1973; Corballis, Kirby, \& Miller, 1972; Corcoran, 1971; Glass, 1984; Townsend, 1971).

Explaining the set-size effect poses a major problem for theorists committed to parallel-access models of shortterm memory retrieval. Although the set-size effect is a natural consequence of a serial search, nonserial models provide no straightforward reason to expect an effect of set size. Complex and ingenious explanations have been formulated, based, for example, on the assumption of declining trace strength of each item in the positive set with increasing set size (e.g., Baddeley \& Ecob, 1973), or a slowing of parallel comparisons as the capacity demanded by multiple comparisons increases (e.g., Townsend, 1971). Although a number of parallel- or directaccess theories can account for the major results of memory-scanning studies, there is little evidence to compel a choice among competing theories or even to show that the serial-search view is wrong.

Interest in the set-size effect has declined as psychology has moved away from the serial conceptions of in- 
formation processing that it seemed to support. Nevertheless, the effect is apparently robust and of potential theoretical importance. The theoretical importance of the set-size effect depends on whether it is the result of a general property of the retrieval of information from shortterm memory, or the result of a process specific to the task in which it is found. Although the set-size effect has been shown to be robust to a variety of parametric changes within Sternberg's basic paradigm, the effects of changes in the structure of the memory-search task itself have not been systematically investigated.

The present study was prompted by the unexpected failure to find a set-size effect in a pilot study similar in design to the present Experiment 1. In the subsequent experiments, the structure of the task employed in Experiment 1 was systematically varied to determine what factors were responsible for the absence of the expected set-size effect.

\section{EXPERIMENT 1}

In Experiment 1, subjects saw series of three to eight digits, the last member of which had occurred previously at some point in the series. The subjects were instructed to respond as soon as any digit was repeated. Trials on which response latency exceeded $1 \mathrm{sec}$ were replaced. Although this task differs from the task employed by Sternberg in several respects, it would seem to require similar access to information in short-term memory. Thus, response latency would be expected to increase with an increase in the number of digits presented before a repetition occurred. The results of the experiment are notable in that no such set-size effect was found.

\section{Method}

Subjects. Six men and 16 women participated in the experiment. The subjects were recruited from psychology classes and in most cases received "bonus points" for their participation. The subjects ranged in age from 18 to 44 years, with a median age of 22.5 years.

Apparatus. The presentation of stimuli, recording of responses, and measurement of response latencies were controlled by an Apple Ile microcomputer. Precise measurement of reaction time was made possible by synchronizing the cathode ray tube raster scan with the controlling program (see Diener \& Smee, 1984, for a discussion). Stimuli were presented on the Apple Monitor II video monitor using the 40-column display. In this display numerals and letters are formed on a $5 \times 7$ pixel grid (approximately $4 \times 6 \mathrm{~mm}$ ). The subjects were allowed to observe the display at the distance they found most comfortable.

Procedure. Each trial consisted of the following sequence of events. A series of three to eight digits was presented sequentially in the center of the screen at the rate of one per second. The last digit in the series was always the only repetition of a digit that had appeared previously in the series. The subjects were instructed to press the " $K$ " key on the computer keyboard with the index finger of the right hand as soon as a digit was displayed that had appeared previously in the series. Once the repeated digit was displayed, it remained on the screen until the subject responded. Thus, after 1 sec had elapsed, temporal cues were available to prompt the response. However, if the response latency was longer than $1 \mathrm{sec}$, the subject was informed by a message on the screen that the response was too slow, and the trial was immediately replaced by another trial with the same series length. If the subject responded at any time prior to $20 \mathrm{msec}$ after the repeated digit had been presented (including false alarms to nonrepeated digits), the message "Anticipation error" was briefly displayed on the screen and the trial was replaced by another of the same series length. Shortly after a response, the subject was instructed by a message on the screen to press the space bar to begin a new trial.

The length of each series and the serial position of the digit that was repeated to end the series were determined by the value of a random number produced by the computer's random (RND) function. The system was programmed such that on any trial the probabilities of series lengths from three to eight digits were equal. Although all series lengths were equally probable on any given trial, the total number of trials at each series length was not set and varied slightly from subject to subject. The serial position of the repeated digit was also selected randomly such that positions from 1 to $n-2$ were equally likely ( $n=$ series length). The last two digits in the series could not be duplicates because, as nothing marked the transition from one digit to the next, consecutive presentations of the same digit would have appeared merely as the presentation of a single digit for a 2 -sec duration.

Each subject received 2 practice trials to clarify the instructions, followed by 160 trials on the task. The subjects were informed by a message on the screen when half of the trials had been completed and were instructed to take a short break if they so desired. The entire procedure took about $30 \mathrm{~min}$.

\section{Results}

Data were originally analyzed for trials on which response latency was less than $1 \mathrm{sec}$. Mean response latencies for the 22 subjects are shown in row 1 of Table 1 for all set sizes. There was no evidence of the expected set-size effect. Response latency actually decreased slightly with set size. The regression of response latency on series length was described by a slope of $-7.31 \mathrm{msec} /$ item $(r=-.89)$ and a zero intercept of $582 \mathrm{msec}$. A subject $\times$ set size analysis of variance (ANOVA) performed on the response-latency data revealed a significant set-size effect $[F(5,105)=2.42$, $p<.05]$. The confidence interval $(95 \%)$ for this slope, derived from the linear component of a subject $\times$ set size trend analysis, was -12.53 to $-2.05 \mathrm{msec} / \mathrm{item}$. Inspection of the mean response latency for each series length reveals that the effect is largely attributable to long latencies for set sizes of two and three digits. For set sizes of four to seven digits, response latency is nearly constant.

Separate subject $\times$ serial position ANOVAs were performed on the response-latency data for each series length to test the effect of the serial position of the repeated digit.

Table 1

Mean Response Latencies (RT) and Error Rates as a Function of Series Length in Experiment 1

\begin{tabular}{lrrrrrr}
\hline & \multicolumn{6}{c}{ Series Length } \\
\cline { 2 - 7 } & \multicolumn{1}{c}{$\mathbf{2}$} & \multicolumn{1}{c}{4} & \multicolumn{1}{c}{5} & \multicolumn{1}{c}{6} & \multicolumn{1}{c}{7} \\
\hline Mean RT < 1 sec & 574.8 & 559.9 & 544.4 & 540.4 & 534.3 & 539.7 \\
Mean RT < 1.5 sec & 595.4 & 575.6 & 559.8 & 559.6 & 550.4 & 566.8 \\
\% RT > I sec* & 4.5 & 4.3 & 4.7 & 6.0 & 8.9 & 9.7 \\
\% Anticipations $\dagger$ & 1.0 & 1.9 & 1.4 & 3.8 & 5.3 & 8.0 \\
\hline
\end{tabular}

*Percentages of responses excluding anticipations. †Percentage of total trials. 
A significant serial-position effect was found only for a set size of $\operatorname{six}[F(4,84)=6.13, p<.001]$. (Because the last digit was never repeated, a set size of six includes only five serial positions; thus $d f=$ set size -2 .) A trend analysis revealed a significant linear trend $[t(84)=2.82$, $p<.01]$, indicating a recency effect. The cubic $[t(84)$ $=3.36, p<.01]$ and quartic $[t(84)=2.29, p<.05]$ trends were also significant.

Although the response latency data subjected to the above-described analyses were based only on responses with latencies under $1 \mathrm{sec}$, long-latency responses were recorded by series length and position of the repeated digit within the series. The proportion of responses with latencies greater than $1 \mathrm{sec}$ was $6.2 \%$. This percentage increased with set size (see Table 1, row 3) at the rate of $1.2 \% /$ item $(r=.93)$. An ANOVA revealed a significant set-size effect $[F(5,105)=3.71, p<.01]$.

Given the present design, it is difficult to determine whether long-latency responses were cued by the recognition of the repeated digit or merely by the recognition that no more digits were forthcoming with the passage of time. It seems unlikely that response latencies under, say, 1,200 msec could have resulted from the subject's responding after detecting that a digit had been displayed for more than $1 \mathrm{sec}$. The average latency of responses requiring more than $1 \mathrm{sec}$ was $2,114 \mathrm{msec}$, compared with the overall average of $549 \mathrm{msec}$ (between-subjects $S D=76.7 \mathrm{msec}$ ) for responses below this cutoff. Examination of the distribution of long-latency responses suggested that responses with latencies in excess of $1.5 \mathrm{sec}$ constituted a different population from responses below that cutoff, although this conclusion was by no means certain.

Although the issue of long-latency responses cannot be resolved satisfactorily, inclusion of long-latency responses in the analysis did not significantly alter the basic findings of the experiment. Mean latencies for each set size were recomputed including all responses with latencies under 1,500 msec (see Table 1, row 2). The slope of the linear regression of the recomputed response latency on set size was $-6.24 \mathrm{msec} /$ item $(r=1.74 ; 95 \%$ confidence interval: -13.51 to $+.97 \mathrm{msec} /$ item), and the zero intercept was $596 \mathrm{msec}$. A subject $\times$ set size ANOVA performed on the recomputed response-latency data revealed no significant effect of set size $[F(5,105)=1.40$, $p>$.05].

The proportions of anticipation errors (i.e., responses preceding the presentation of the repeated digit) are shown in row 4 of Table 1 for each series length. The percentage of anticipation errors, averaged across series length, was $3.56 \%$. The trend for this proportion to increase with series length was confirmed by an ANOVA $[F(5,105)=$ $27.8, p<.001]$. A linear regression of the mean proportion of anticipation errors on series length yielded a slope of $.013 /$ item and a correlation of $r=.93$. This analysis is compatible with the proposition that the tendency to respond prematurely was constant for each unit of time and increased only as a function of the increasing total time with increasing series length. ${ }^{1}$

\section{Discussion}

The important result of Experiment 1 is the failure of response latency to increase with series length. The small tendency for response latency to actually decrease with set size seems attributable to surprise or a failure of preparation on the part of the subjects when a digit was repeated early in the series. The present task differs from Sternberg's task in several respects, any of which might be responsible for the absence of a set-size effect. In the current task:

1. Subjects eventually made a positive response on all the trials rather than on half the trials. Moreover, because the probabilities of all six series lengths were equal, the probability of responding after the second digit was presented was $1 \%$, after three digits (assuming no response was made to the second) was $1 / 5$, and so on. In Sternberg's task, the probability of a positive response to any probe digit can be kept constant across set size at $1 / 2$, or any value selected. Increasing the probability of a positive response has been found to cause a decrease in the latency of positive responses on Sternberg's task (Sternberg, 1969).

2. The digit preceding the subject's response was not specifically identified as a probe. Subjects could know that a response was required only after recognizing that the digit had occurred previously in the series.

3 . Because the test digit was not identified, a decision was required following the presentation of each item in the memory set (after the first two digits). In contrast, Sternberg's task requires only that a single decision be made upon presentation of the test digit.

4. The repeated digit immediately followed the last new digit in the series. Even if the repeated digit were specifically identified as the memory probe, there would be no period to prepare for a response as in Sternberg's task.

5. Subjects responded with a single key rather than choosing between one key for positive responses and a second for negative responses.

\section{EXPERIMENT 2}

Experiments 2-4 were undertaken to investigate the role of the above factors in the absence of a set-size effect in Experiment 1. Experiment 2 was similar to Experiment 1 except that on half of the trials in Experiment 2, no digit was repeated and no explicit response was required (item 1 above).

\section{Method}

Subjects. Twenty subjects ( 8 men and 12 women) participated in the experiment. The subjects were recruited from a number of courses in psychology and education. Four of the subjects had participated previously in one of the author's memory-scanning studies. The subjects ranged in age from 19 to 50 years, with a median age of 28.5 years.

Apparatus and Procedure. The apparatus and procedure employed in Experiment 2 were the same as in Experiment 1, except that on half of the trials at each series length the last digit in the series had not been presented previously in the series. On these "negative" trials, the last digit in the series was displayed for 
$1.5 \mathrm{sec}$, then disappeared. The subjects were informed of this procedure and instructed to press the " $K$ " key on the computer keyboard if a digit appeared that had been displayed previously in the series and to do nothing otherwise. On positive trials, the test digit was displayed until either a response was recorded or $1.5 \mathrm{sec}$ had elapsed. If the subject failed to respond within $1 \mathrm{sec}$ of the presentation of a positive probe, the message "Response time too long" was displayed on the screen immediately after the subject's response. However, data were saved for all trials on which response latency was less than $1.5 \mathrm{sec}$. If the subject responded after a negative probe had been presented, the message "You should not have responded" appeared on the screen. If the subject responded prior to $25 \mathrm{msec}$ after the presentation of the test digit, the message "Anticipation error" was displayed. In the event of any of these errors, the trial was replaced by another of the same series length.

\section{Results}

Data were first analyzed for responses with latencies less than $1 \mathrm{sec}$. Mean latencies of these responses for each set size are displayed in row 1 of Table 2. There was no linear increase in response latency with an increase in series length. The slope of the linear regression of response latency on set size was $.81 \mathrm{msec} / \mathrm{item}(r=.12 ; 95 \%$ confidence interval: -2.98 to $4.61 \mathrm{msec} /$ item), and the zero intercept was $657 \mathrm{msec}$. An ANOVA revealed a significant set-size effect $[F(5,95)=2.55, p<.05]$. A trend analysis revealed only a significant quadratic trend $[t(95)$ $=3.54, p<.001$ ], confirming the tendency apparent in the data for the shortest response latencies to occur at the middle series lengths.

As in Experiment 1, including longer-latency responses in the analysis had little effect on the pattern of results. Mean response latencies recomputed to include all responses (no response latency could exceed $1.5 \mathrm{sec}$ ) are shown in row 2 of Table 2 , as a function of set size. The regression of the reanalyzed response latency on set size was described by a slope of $1.19 \mathrm{msec} /$ item $(r=.11 ; 95 \%$ confidence interval: -5.58 to 7.94 ) and an intercept of $702 \mathrm{msec}$. An ANOVA revealed no significant set-size effect $[F(5,95)=1.92, p>.05]$, although the quadratic component of a trend analysis was significant $[t(95)=$ $2.27, p<.05]$.

Responses with latencies in excess of $1 \mathrm{sec}$ accounted for $9.0 \%$ of the total responses. The proportion of responses with latencies in excess of $1 \mathrm{sec}$ (Table 2, row 3 ) did not increase significantly with set size. The slope of the regression of the proportion of long responses

Table 2

Mean Response Latencies (RT) and Error Rates as a Function of Series Length in Experiment 2

\begin{tabular}{lrrrrrr}
\hline & \multicolumn{6}{c}{ Series Length } \\
\cline { 2 - 7 } & \multicolumn{1}{c}{2} & \multicolumn{1}{c}{3} & \multicolumn{1}{c}{4} & \multicolumn{1}{c}{5} & \multicolumn{1}{c}{6} & \multicolumn{1}{c}{7} \\
\hline Mean RT < 1 sec & 675.0 & 656.2 & 647.5 & 649.6 & 660.3 & 677.8 \\
Mean RT < 1.5 sec & 727.0 & 686.5 & 711.7 & 680.1 & 712.9 & 725.7 \\
\% RT > 1 sec* & 10.5 & 6.0 & 10.4 & 6.4 & 10.8 & 10.0 \\
\% No Response† & .3 & 2.6 & 6.5 & 10.3 & 12.4 & 9.7 \\
\% Anticipations $\ddagger$ & .5 & 1.8 & 1.9 & 2.3 & 4.1 & 8.1 \\
\% False Alarms\$ & .7 & .7 & 1.4 & 2.4 & 3.8 & 11.9 \\
\hline
\end{tabular}

*Percentage of correct responses. †Percentage of positive trials. $\quad \ddagger$ Percentage of total trials. §Percentage of negative trials. on set size was $.22 \% /$ item $(r=.19)$. An ANOVA performed on the proportion of long-latency reponses revealed no significant set-size effect $[F(5,95)=1.79$, $p>.05]$

The subjects failed to respond within the 1.5 -sec deadline on $6.97 \%$ of the positive trials. Although this proportion (Table 2, row 4) appeared to increase with set size $(2.28 \% /$ item; $r=.91)$, an ANOVA revealed no significant set-size effect $[F(5,95)=1.50, p>.05]$.

Separate subject $\times$ serial position ANOVAs were performed on the response latency data for set sizes three to seven. For responses with latencies below $1 \mathrm{sec}$, there was a significant serial-position effect only for a set size of seven digits $[F(5,91)=3.33, p<.01]$. This effect was attributable to short response latencies for serial positions two and three. None of the components of a trend analysis was significant. When longer-latency responses were included in the data, there were no significant serialposition effects at any set size.

Anticipation errors (responses occurring before $25 \mathrm{msec}$ after the presentation of the final digit) occurred on $3.1 \%$ of the trials. This proportion (Table 2, row 5) increased with set size at the rate of $1.28 \% /$ item $(r=.90)$. This setsize effect was significant $[F(5,95)=5.16, p<.001]$.

The subjects responded incorrectly on $3.48 \%$ of the negative trials (i.e., more than $25 \mathrm{msec}$ after the final digit had been presented on negative trials). The proportion of incorrect responses on negative trials ("\% False Alarms," Table 2, row 6) increased as a function of set size at the rate of $1.89 \% /$ item $(r=.82)$. An ANOVA performed on the proportion of incorrect responses revealed a significant set-size effect $[F(5,95)=10.37, p<.001]$. Because anticipation errors are excluded, the increase in incorrect responses with increasing set size is not attributable to a greater opportunity for false alarms as the series lengthens. The increase in false alarms would seem to indicate either increased expectancy of a positive digit or a lowered response criterion (in the signal detection sense) with increasing set size.

\section{Discussion}

The most striking result of including negative trials in the design was the increase in overall response latency. Including all responses with latencies under $1.5 \mathrm{sec}$, the overall mean response latency in Experiment 2 $(707 \mathrm{msec})$ was $141 \mathrm{msec}$ greater than the overall mean response latency in Experiment $1(566 \mathrm{msec})$. This effect is somewhat surprising when one considers that the objective probability of a repeated digit at the third position in the series in Experiment 1 was 1:6, whereas the probability of a repeated digit following the seventh digit in Experiment 2 was $1: 2$. If response latency were determined primarily by response probability, the latency of responses for the longer series lengths in Experiment 2 should be closer to the values for short series lengths in Experiment 1. Apparently, deciding when to respond, as in Experiment 1, requires considerably less time than deciding whether to respond, as in Experiment 2. 
The quadratic set-size effect in Experiment 2 is difficult to interpret. This effect could be the result of a tendency for maximum preparedness to respond at the average series length. It seems unlikely, however, that preparedness to respond would wane as series length increased. The increase in false alarms at the longest series lengths suggests that preparedness to respond increased for the longer series. It is possible that the quadratic effect is the result of two competing tendencies: a lack of preparedness to respond when a digit is repeated early in the series (as in Experiment 1), and a tendency for response latency to increase for the larger set sizes, from some other source.

\section{EXPERIMENT 3}

Regardless of its source, the set-size effect in Experiment 2 is of neither the nature nor the magnitude of setsize effects usually reported in memory-scanning studies. A major difference between the tasks employed in Experiments 1 and 2 and the typical memory-search task concerns the designation of the test digit. In Experiments 1 and 2 the test digit could be identified as a test digit only after the subject determined that the digit had occurred previously in the series. In the usual Sternberg task, the test stimulus is designated independently of its membership in the positive set. Experiment 3 was designed to be as similar as possible to Experiment 2, except that the test digit was explicitly designated.

\begin{abstract}
Method
Subjects. Twenty subjects ( 7 men and 13 women) participated in the experiment. The subjects were recruited from a subject pool similar to those of the previous experiments. The subjects ranged in age from 16 to 38 years with a median age of 23.5 years.

Apparatus and Procedure. The apparatus and procedures employed in Experiment 3 were the same as in Experiment 2, with the following exception: The last digit in the series was presented in an inverse format; that is, the numerals were formed by dark areas in an otherwise illuminated $5 \times 7$ pixel grid. As in Experiment 2, on half of the trials at each series length, the test digit had been displayed previously in the series. Except that the subjects were informed that the test digit would be displayed in an inverse format, all instructions to the subjects and all error messages were the same as in Experiment 2.
\end{abstract}

\section{Results}

Data were originally analyzed for responses with latencies less than $1 \mathrm{sec}$. Mean latencies for responses of less than $1 \mathrm{sec}$ are displayed in Table 3, row 1. Response latency did not increase with series length. The slope of the linear regression of response latency on set size was $.77 \mathrm{msec} / \mathrm{item}(r=.193 ; 95 \%$ confidence interval: -4.52 to $2.99 \mathrm{msec} /$ item), and the zero intercept was $672 \mathrm{msec}$. An ANOVA revealed no significant set-size effect $[F(5,95)<1]$.

When the data were reanalyzed to include all responses, a somewhat different picture emerged. The recomputed mean latencies are shown in row 2 of Table 3 . The regression of the reanalyzed response latency on set size was
Table 3

Mean Response Latencies (RT) and Error Rates as a

Function of Series Length in Experiment 3

\begin{tabular}{lrrrrrr}
\hline & \multicolumn{6}{c}{ Series Length } \\
\cline { 2 - 7 } & \multicolumn{1}{c}{2} & \multicolumn{1}{c}{4} & \multicolumn{1}{c}{5} & \multicolumn{1}{c}{6} & \multicolumn{1}{c}{7} \\
\hline Mean RT < 1 sec & 677.1 & 660.2 & 669.5 & 671.9 & 658.4 & 672.2 \\
Mean RT < 1.5 sec & 702.1 & 665.9 & 701.3 & 704.7 & 734.0 & 724.8 \\
\% RT > 1 sec* & 6.0 & 1.8 & 7.1 & 7.1 & 13.1 & 9.9 \\
\% No Response $\dagger$ & 0.0 & 1.9 & 3.7 & 6.0 & 6.7 & 8.1 \\
\%False Alarms $\ddagger$ & 5.7 & 6.0 & 5.7 & 11.9 & 11.0 & 14.4 \\
\hline
\end{tabular}

*Percentage of correct responses. †Percentage of positive trials. $\ddagger$ Percentage of negative trials.

described by a slope of $9.17 \mathrm{msec} / \mathrm{item}(r=.727 ; 95 \%$ confidence interval: 3.77 to 14.57 ) and an intercept of $664 \mathrm{msec}$. An ANOVA revealed a significant set-size effect $[F(5,95)=4.28, p<.01]$, and a trend analysis revealed significant linear $[t(95)=3.37, p<.01]$ and cubic $[t(95)=-2.47, p<.05]$ trends.

Inspection of the means of the inclusive data for the different set sizes reveals that the latencies for set sizes of two, four, and five digits are nearly equal. The mean latency for a set size of three digits is some $37 \mathrm{msec}$ lower than these values, due largely to the small proportion of responses with latencies in excess of $1 \mathrm{sec}$. If this point is regarded as anomalous, the major effect of including long-latency responses in the analysis is the relative increase in response latency for the two largest set sizes. On the other hand, it is possible that the short response latency for a set size of three digits represents a real trend and that the longer latency for a set size of two digits is anomalous.

Responses with latencies in excess of $1 \mathrm{sec}$ accounted for $7.50 \%$ of the total responses. The proportion of responses with latencies in excess of $1 \mathrm{sec}$ increased as a function of set size (see Table 3, row 3). An ANOVA performed on the proportion of long-latency responses revealed a significant set-size effect $[F(5,95)=7.17$, $p<.001]$. The regression of the proportion of longlatency responses on set size was described by a slope of $1.52 \% /$ item $(r=.75)$.

The mean of the long-latency responses was $1,182 \mathrm{msec}$. Although the proportion of long-latency responses increased with set size, there was no significant increase in the latency of these responses. The slope of the regression of the latency of the long-latency responses on set size was $9.09 \mathrm{msec} /$ item $(r=.37)$. A subject $\times$ set size ANOVA (with many data points missing) performed on the latency of the long-latency responses revealed no significant set-size effect $[F(5,38)<1]$. Thus, the small set-size effect apparent in the combined data is attributable to an increase in the number (rather than the latency) of long-latency responses with increasing set-size.

The subjects failed to respond within the 1.5 -sec deadline on $4.40 \%$ of the positive trials. This proportion (shown in Table 3, row 4) increased with set size $(1.63 \% /$ item; $r=.99)$. An ANOVA performed on the 
proportion revealed a significant set-size effect $[F(5,95)$ $=3.05, p<.05]$.

Separate subject $\times$ serial position ANOVAs were performed on the response-latency data for set sizes three to seven. For responses with latencies below $1 \mathrm{sec}$, there were no significant serial-position effects for any set size. When longer-latency responses were included in the analysis, a significant serial-position effect was found only for a set size of five $[F(3,60)=4.15, p<.01]$. A trend analysis revealed only a significant linear trend $[t(60)=$ $2.64, p<.05$ ], indicating a recency effect.

The subjects responded incorrectly on $9.20 \%$ of the negative trials. The proportion of incorrect responses on negative trials (shown in Table 3 , row 5 ) increased as a function of set size at the rate of $1.86 \% /$ item $(r=.91)$. An ANOVA revealed a significant set-size effect $[F(5,95)$ $=3.75, p<.01]$.

Only 20 anticipation errors (responses occurring before $25 \mathrm{msec}$ after the presentation of the inverse digit) were recorded. This figure represents only $.56 \%$ of the total trials. Because of the small number of these errors, no further analysis was performed.

\section{Discussion}

The explicit identification of the test stimulus in Experiment 3 would seem to change the nature of the subjects' task from that of Experiment 2 in two major ways. First, the subject can identify the test digit before knowing whether it has been previously presented. This might be expected to alter the nature of the decision process. Second, because the test digit is explicitly designated, the subject is not forced to make a decision for each digit that is presented. This might be expected to affect the trace strength of items in the series. Given the apparent differences in processing requirements, it is surprising that the subjects' performance in Experiments 2 and 3 was so similar. Including all responses in the analysis, the mean response latency for all set sizes in Experiment 3 was $705 \mathrm{msec}$, compared with $707 \mathrm{msec}$ in Experiment 2.

Despite the similarities in the data from Experiments 2 and 3, a number of differences in the pattern of results from the two experiments are worthy of discussion. In Experiment 2, the shortest mean latencies (for the shortlatency responses) occurred at set sizes of four and five digits. This effect was not evident in Experiment 3. Given the fact that the short-latency means for all other set sizes in Experiment 3 were within $6 \mathrm{msec}$ of the value for the same set size in Experiment 2, the 22-msec increases in the mean latencies for set sizes of four and five, relative to those in Experiment 2, are difficult to understand.

Several differences in the results of Experiments 2 and 3 are easily understandable as consequences of explicit designation of the test digit. The decrease in anticipation errors in Experiment 3 would be expected because the subject knows that a response is required only in the case of a clearly identified test digit. As evidenced by the ratios of misses (i.e., no responses to positive probes) to false alarms (i.e., responses to negative probes), the sub- jects of Experiment 2 were more conservative in responding than were the subjects of Experiment 3. Averaged across set size, the percentage of misses in Experiment 2 was $6.97 \%$ and the percentage of false alarms was $3.48 \%$ $(\beta=1.74)$ compared with misses of $4.40 \%$ and false alarms of $9.10 \%(\beta=.57)$ in Experiment 3. A more liberal response criterion would be expected in Experiment 3 because the subject knows that the objective probability of a positive test digit is 50\%. In Experiment 2, the probability of a repeated digit varied with series length and was lower than $50 \%$ for all but the longest series length.

The proportion of long-latency responses in Experiment 3 increased with set size. As a consequence, including long-latency responses in the data resulted in an increase in response latency with increasing set size. This finding might be explained by reference to a modified version of the trace-strength theory presented by Baddeley and Ecob (1973), who assumed that subjects have immediate access to memory loci corresponding to all potential members of the positive set (e.g., all digits 0-9). Presentation of the positive set results in the temporary activation of the subset of these loci corresponding to the positive set. Subjects base their decision about the membership of the test stimulus in the positive set on the level of activation (the trace strength) of the locus corresponding to the test, performing the equivalent of a signal detection analysis. The time necessary to complete this analysis increases as the difference between the level of activation of the locus corresponding to the test digit and an adjustable response criterion decreases.

To account for the set-size effect, Baddeley and Ecob (1973) assumed that a fixed quantity of trace strength is divided among all members of the positive set. Thus, the larger the memory set, the smaller the difference between the level of activation of the members of the positive set and potential distractors. For present purposes, however, let us assume that the trace strength of well-rehearsed members of the memory set is independent of set size. However, as the number of items in the positive set increases, the proportion of trials on which the test digit is at low trace strength, due to the failure of attention or rehearsal, increases. Thus, the long-latency responses in Experiment 3 correspond to items that, by virtue of unusually low trace strength, are close to the subject's response criterion. These assumptions would account for the absence of a set-size effect for short-latency responses and the presence of a small set-size effect when longlatency responses are included in the analysis, as well as for the increases in both misses and false alarms with in creasing set size.

If the foregoing analysis is correct, an increase in the proportion of long-latency responses with increasing set size might have been expected in Experiment 2. Comparison of the proportions of long-latency responses as a function of set size in Experiments 2 and 3 reveals that the difference between the pattern of results in the two experiments is attributable primarily to fewer long-latency responses for smaller set sizes in Experiment 3. It seems 
reasonable to conclude that the larger proportion of longlatency responses for the shorter set sizes in Experiment 2 is the result of the subjects' lack of preparedness to respond (at least on some proportion of the trials) when a digit was repeated early in the series. Explicit designation of the test digit in Experiment 3 might be expected to reduce this effect.

\section{EXPERIMENT 4}

Although the set-size effect in Experiment 3 is interpretable, it is clearly not of the magnitude usually encountered in memory-scanning tasks. The task employed in Experiment 3 differed from the usual Sternberg task in that (1) no explicit negative response was required and (2) the test digit followed the presentation of the last digit in the series immediately, with no warning that the next digit to be presented would be the test digit. The absence of the usual set-size effect in Experiment 3 might be attributable to either of these differences between the present task and the more common paradigm.

Egeth, Marcus, and Bevan (1972) investigated the effects of an explicit negative response on the memoryscanning task. They reported that the set-size effect was eliminated by the absence of an explicit negative response when the elements of the memory set formed meaningful, familiar units (e.g., "1, 2, 3"). When the elements of the memory set did not form meaningful units (e.g., " $1,4,7$ "), the usual set-size effect was found, despite elimination of the explicit negative response. Egeth et al.'s study was unusual in some respects: Only set sizes of one and three digits were used in the study, and the digit " 1 " always served as the one-digit set. Nevertheless, the study provided evidence that the absence of an explicit negative response does not, by itself, eliminate the set-size effect. This evidence prompted an investigation of the alternative possibility that the absence of a warning period was responsible for the lack of the usual set-size effect in the present experiments.

\section{Method}

Subjects. Twenty subjects ( 6 men and 14 women), recruited from a subject pool similar to those of the previous experiments, participated in Experiment 4. The subjects ranged in age from 19 to 41 years with a median age of 27.5 years.

Procedure. The procedures employed in Experiment 4 differed from those of Experiment 3 only by the insertion of a $1-\mathrm{sec}$ interval between the presentation of the last digit in the series and the inverse probe digit. This interval was marked by an illuminated square (an inverse space) at which the digits comprising the memory set appeared.

\section{Results}

Following the pattern established in the previous experiments, data were analyzed first for responses with latencies less than $1 \mathrm{sec}$, and then reanalyzed including longer-latency responses. Mean response latencies at each series length are displayed in Table 4, row 1. For responses with latencies below the 1-sec cutoff, the slope of the regression of response latency on set size was
Table 4

Mean Response Latencies (RT) and Error Rates as a Function of Series Length in Experiment 4

Series Length

\begin{tabular}{rrrrrr}
\multicolumn{7}{c}{ Series Length } \\
\hline \multicolumn{1}{c}{3} & \multicolumn{1}{c}{4} & \multicolumn{1}{c}{5} & \multicolumn{1}{c}{6} & \multicolumn{1}{c}{7} \\
\hline 469.6 & 501.1 & 541.5 & 551.4 & 579.9 & 574.0 \\
488.4 & 514.3 & 556.0 & 572.1 & 651.9 & 616.3 \\
2.3 & 1.7 & 1.4 & 2.7 & 7.6 & 5.3 \\
0.3 & 1.8 & 1.7 & 4.0 & 5.3 & 6.5 \\
3.3 & 1.8 & 5.0 & 6.0 & 9.2 & 11.9
\end{tabular}

*Percentage of correct responses. †Percentage of positive trials. $\quad \Varangle$ Percentage of negative trials.

$21.95 \mathrm{msec} / \mathrm{item}(r=.95 ; 95 \%$ confidence interval 18.18 to $25.72 \mathrm{msec} / \mathrm{item}$ ) and the zero intercept was $437 \mathrm{msec}$. An ANOVA revealed a significant set-size effect $[F(5,95)$ $=29.38, p<.001]$. Including responses with latencies over $1 \mathrm{sec}$ resulted in a slope of $30.53 \mathrm{msec} / \mathrm{item}(r=.93$; $95 \%$ confidence interval: 23.70 to 37.29 ) and an intercept of $429 \mathrm{msec}$. The set-size effect for the inclusive data was significant $[F(5,95)=18.10, p<.001]$.

Responses with latencies over $1 \mathrm{sec}$ accounted for $3.5 \%$ of the total responses. The proportion of responses with latencies in excess of $1 \mathrm{sec}$ (Table 4, row 3) increased with set size at the rate of $.97 \% /$ item $(r=.74)$. This increase with set size was significant $[F(5,95)=3.30$, $p<.01]$. The mean latency of the long-latency responses was $1,194 \mathrm{msec}$, quite similar to the comparable value $(1,181 \mathrm{msec})$ in Experiment 3. The latency of these responses appeared to decrease with set size (slope $-24.02 \mathrm{msec} / \mathrm{item}, r=-.623$ ). However, a subject $\times$ set size ANOVA performed on the latency of the long-latency responses (with many missing data points) revealed no significant set-size effect $[F(5,38)<1]$.

The subjects failed to respond within the 1.5 -sec deadline on $3.3 \%$ of the positive trials. This proportion (Table 4, row 4) increased with set size at the rate of $1.25 \% /$ item $(r=.98)$. However, an ANOVA revealed no significant set-size effect $[F(5,95)=2.13, p>.05]$.

Separate subject $\times$ serial position ANOVAs were performed on the response latency data for set sizes three to seven. For responses with latencies below $1 \mathrm{sec}$, there was a significant serial-position (recency) effect only for a set size of three digits $[F(1,19)=4.53, p<.05]$. Including longer-latency responses in the data resulted in a significant serial-position effect only for a set size of four $[F(2,38)=3.10, p<.05]$. A trend analysis revealed only a significant quadratic trend $[t(38)=2.32, p<.05]$, confirming a primacy-recency effect.

The subjects responded incorrectly on $6.20 \%$ of the negative trials. The proportion of incorrect responses on negative trials (Table 4 , row 5 ) increased significantly $[F(5,95)=5.05, p<.001]$ as a function of set size at the rate of $1.89 \% /$ item $(r=.91)$.

Only 12 anticipation errors (.35\% of the total trials) were recorded. Because of the small number of these errors, no further analysis was performed.

To confirm the differences between the results of Experiments 3 and 4 , a condition $\times$ set size $\times$ subject 
within-condition ANOVA was performed on the shortlatency data from the two experiments. A significant $[F(1,38)=57.65, p<.001]$ condition effect confirmed the lower overall response latency in Experiment 4, and a significant set size $\times$ condition interaction $[F(5,190)$ $=16.83, p<.001]$ confirmed the greater set-size effect.

\section{Discussion}

The addition of a warning period preceding the test digit in Experiment 4 had several sizable effects. First, overall response latency was greatly reduced relative to $\mathbf{E x}$ periments 2 and 3 and was lower even than in Experiment 1 . The mean response latency across all series lengths in Experiment 4 was $566 \mathrm{msec}$, compared with $705 \mathrm{msec}$ in Experiment 3 (all responses included in both cases). Moreover, the lowest mean response latency for any set size in Experiment 3 was longer than the longest mean latency for any set size in Experiment 4 . Second, error rates of all kinds in Experiment 4 were lower than the comparable values in the previous experiments. Third, the slope of the response-latency function was considerably greater in Experiment 4 than in Experiment 3 when all responses were included, and was fairly large for the shorter-latency responses.

A rather cursory examination of the literature reveals that most memory-scanning studies do not report excluding any responses from analysis on the basis of long latencies. No cases were found in which response latencies of less than $1.5 \mathrm{sec}$ were excluded. Thus, the inclusive data in the present study provides the appropriate basis for comparisons with other results. The 30.5- $\mathrm{msec} / \mathrm{item}$ slope of the response-latency function in Experiment 4 (including long-latency responses) is only slightly lower than the slopes typically reported on Sternberg's task. Cavanagh (1972) reported $33.4 \mathrm{msec} /$ item as the average slope for eight studies using digits on the scanning task. Studies conducted in my laboratory, using the same equipment and similar subjects as in the present study on the two-response Sternberg task, typically yield slopes for combined positive and negative responses of $30-35 \mathrm{msec} / \mathrm{item}$.

It is difficult to see how the trace-strength analysis applied to the results of Experiments 2 and 3 might be extended to account for the increase in the slope of the response-latency function associated with the introduction of the preprobe delay in Experiment 4. Although, in the absence of evidence to the contrary, it might be argued that the trace strength associated with the elements of different sized sets declines differentially over the delay interval, the fact that overall response latency in Experiment 4 was shorter than in Experiment 3, despite lower error rates, is not interpretable by reference to decaying trace strength.

Although the trace-strength mechanism invoked to account for the results of Experiment 3 seems unlikely to account for the increase in the slope of the responselatency function in Experiment 4, a similar effect is apparent in the data. The inclusion of long-latency responses in Experiment 4 resulted in an increase in the slope of the response-latency function of $8.55 \mathrm{msec} / \mathrm{item}$, only slightly lower than the increase of $9.94 \mathrm{msec} /$ item resulting from the inclusion of long-latency responses in Experiment 3.

\section{GENERAL DISCUSSION}

The present results seem to indicate that a preprobe delay interval is necessary for producing the typical set-size effect, at least in tasks that require no explicit negative response. Although the preprobe delay has apparently not been entirely eliminated in previous memory-scanning studies, the duration of the delay period has been varied. Unfortunately for present purposes, studies in which the preprobe delay has been manipulated have usually been concerned with serial-position rather than set-size effects. As a consequence, a single set size has often been used (e.g., Corballis, 1967; Morin, De Rosa, \& Stultz, 1967), or the preprobe delay has been confounded with presentation rate (e.g., Burrows \& Okada, 1971).

Available data indicate that, within a fairly wide range, the duration of the preprobe delay has little effect on the slope of the response-latency function. Clifton and Birenbaum (1970) presented one to seven digits at a rate of $1.5 \mathrm{sec} /$ digit. Three different durations were used for the preprobe delay interval: $.8,2.8$, and $4.5 \mathrm{sec}$. There was no significant set size $\times$ delay interaction for either positive or negative responses. It seems possible that there is a minimum value above which the duration of the preprobe delay has little effect.

Aubé and Murdock (1974) presented one to eight digits at the rate of $250 \mathrm{msec} /$ digit followed by a preprobe delay of $300 \mathrm{msec}$. The slope of the response latency function (computed from the published data by Cavanagh, 1976) was $23 \mathrm{msec} / \mathrm{item}$ for positive responses and $16 \mathrm{msec} / \mathrm{item}$ for negative responses. Although these slopes are lower than those typically reported in memoryscanning studies, the effect might be attributed to the fast presentation rate, or the short delay, or both.

Several possible explanations for the absence of a setsize effect in the absence of a preprobe delay might be considered:

1. The absence of a preprobe delay might somehow mask the increased retrieval time that results from increasing the set size. For example, as the series lengthens, the subjects' expectation that a test digit will be presented might rise. The reduction in response latency associated with this increased expectancy might offset increased retrieval time for larger sets (see Nickerson, 1967, for a discussion of expectancy effects). A preprobe delay might serve to control for expectancy effects by providing a period during which the subject knows that the next item will serve as a probe.

Although expectancy effects might account for the small decrease in response latency with increasing set size in Experiment 1, it is difficult to see how the results of the subsequent experiments might be explained in terms of expectancy. The addition of negative trials and the ex- 
plicit identification of the test digit in Experiments 2 and 3 , if not sufficient to eliminate expectancy effects, should have reduced them sufficiently to reveal at least a portion of the usual set-size effect. Moreover, the nearly zeroslope functions of Experiments 2 and 3 would impose the unlikely requirement of expectancy effects of almost exactly the magnitude of the countervailing retrieval-time effects.

2. A preprobe delay might be necessary to prepare the memory set for the search process. In the absence of the delay, the memory set may not be stored in a form that is amenable to the search process that results in the typical set-size effect. It does not seem likely that the increase in the slope of the response-latency function with the introduction of a preprobe delay is the consequence of retrieving the positive set from inactive memory during the delay interval. Intervening tasks imposed between the presentation of the memory set and presentation of the probe, which presumably remove the positive set from short-term memory (e.g., Sternberg, 1969), have been found to increase rather than decrease the slope of the response-latency function.

The fact that the introduction of the preprobe delay resulted in lower response latency across all set sizes would seem to rule out trace-strength decay as the primary mechanism whereby the delay interval might contribute to the set-size effect. It is conceivable that rehearsal of the set during the delay interval might raise the trace strength of small sets relative to that of larger sets. However, it is difficult to see how a two-digit set might increase appreciably in trace strength as a consequence of an additional second of rehearsal. Moreover, the reduction in average response latency resulting from the introduction of the preprobe delay seems too large to result from changes in trace strength.

3. The effect of the preprobe delay might be attributable to active preparation for a response during the delay interval. This preparation to respond might result in decreased overall response latency by lowering the recognition threshold for members of the postive set, or by decreasing decision time once a positive probe is recognized. The increased slope of the response-latency function resulting from the addition of the preprobe delay might be the consequence of more effective preparation for small sets than for large sets. For a set size of a single digit, preparation might be quite effective: "respond if ' 5 ,' else don't respond." As the number of digits in the positive set increases, improvement in response latency relative to what would be possible in the absence of a warning period might diminish.

The response-preparation hypothesis would seem to give an adequate account of the results of the present study and does not seem incompatible with the major findings of studies using Sternberg's memory-search task. The explanation is, of course, far from complete. It is not clear how preparation during the preprobe delay might reduce response latency once the test digit is presented. There is no reason to expect the set-size effects resulting from response preparation to be linear. Although the theory could be elaborated to account for the linear nature of the set-size effect and other phenomena found in memoryscanning studies, such theorizing is probably premature until the major premise is more adequately tested and a number of empirical questions are addressed.

The effect of eliminating the preprobe delay from a memory-scanning task requiring an explicit negative response should clearly be investigated. The addition of an explicit negative response might well alter the responselatency distributions. It is possible that if an explicit negative response had been available in the present study, subjects might have given incorrect negative responses to some of the items that produced long-latency positive responses. Systematic investigation of the effects of very short preprobe delays might also prove informative. Knowledge of the minimum delay interval necessary to produce a set-size effect of the typical magnitude would limit the kinds of processes that might be supposed to occur during the delay interval.

It would also be informative to examine the entire response-latency distributions of individual subjects in tasks with and without preprobe delays. A detailed examination of these distributions would provide a better picture of the role of long-latency responses in the setsize effect. Unfortunately, in the present study, only the means for the various set sizes and serial positions were recorded for response latencies under $1 \mathrm{sec}$. A finegrained analysis of the response-latency distribution is therefore impossible.

Regardless of the outcome of future empirical and theoretical work, the present study suggests that the set-size effect is a less general phenomenon than has been typically assumed. The failure to find the usual set-size effect in the absence of a preprobe delay raises doubts with respect to the widely held assumption that the set-size effect results from greater time to access the elements of short-term memory as the size of the memory set increases.

\section{REFERENCES}

AubÉ, M., \& MuRDock, B. (1974). Sensory stores and high-speed scanning. Memory \& Cognition, 2, 27-33.

Baddeley, A. D., \& ECOB, J. R. (1973). Reaction time and short-term memory: Implications of repetition effects for the high-speed exhaustive scan hypothesis. Quarterly Journal of Experimental Psychology, 25, 229-240.

Burrows, D. , \& OKADA, R. (1971). Serial position effects in high-speed memory search. Perception \& Psychophysics, 10, 305-308.

CaVanagh, J. P. (1972). Relation between the immediate memory span and the memory search rate. Psychological Review, 79, 525-530.

Cavanagh, J. P. (1976). Holographic and trace strength models of rehearsal effects in the item recognition task. Memory \& Cognition, 4 , 186-199.

Clifton, C., \&irenbaum, S. (1970). Effects of serial position and delay of probe in a memory scan task. Journal of Experimental Psychology, 86, 69-76.

Corballis, M. C. (1967). Serial order in recognition and recall. Journal of Experimental Psychology, 74, 99-105.

Corballis, M. C., Kirby, J., \& Miller, A. (1972). Access to ele- 
ments of a memorized list. Joumal of Experimental Psychology, 94, 185-190.

Corcoran, D. W. J. (1971). Pattern recognition. Harmondsworth, England: Penguin Books.

Diener, D., \& SMEE, W. P. (1984). Apple tachistoscope. Behavior Research Methods, Instruments, \& Computers, 16, 540-544.

Egeth, H., Marcus, N., \& Bevan, W. (1972). Target-set and responseset interactional: Implications for models of human information processing. Science, 176, 1447-1448.

GLAss, A. L. (1984). Effect of memory set on reaction time. In J. R. Anderson \& S. M. Kosslyn (Eds.), Tutorials in learming and memory (pp. 119-136). San Francisco: W. H. Freeman.

How ARD, J. H. (1975). The attentional demands of negation in a memory-scanning task. Memory \& Cognition, 3, 319-324.

Krueger, L. E. (1975). The effect of an added memory set on item recognition: A test of parallel-dependent vs. serial-comparison models. Memory \& Cognition, 3, 485-495.

Morin, R. E., De Rosa, D. V., \& Stultz, V. (1967). Recognition memory and reaction time. Acta Psychologica, 27, 298-305.

NICKERSON, R. S. (1967). Expectancy, waiting time and the psychological refractory period. Acta Psychologica, 27, 23-34.

STERNBERG, S. (1966). High-speed scanning in human memory. Science, $153,652-654$

STERNBERG, S. (1969). Memory scanning: Mental processes revealed by reaction-time experiments. American Scientist, 57, 421-457.
Sternberg, S. (1975). Memory scanning: New findings and current controversies. Quarterly Journal of Experimental Psychology, 27, 1-32.

TownSEND, J. T. (1971). A note on the identifiability of parallel and serial processes. Perception \& Psychophysics, 10, 161-163.

\section{NOTE}

1. If the probability ( $p$ ) of a response during an arbitrary time interval remains stable during $n$ of these intervals, the probability of a response occurring at some time during the $n$ intervals $(P)$ is the complement of the probability that no response occurs during any of the $\boldsymbol{n}$ intervals:

$$
P=1-(1-p)^{n} \text {. }
$$

If $p$ is small enough that $p^{2}$ and higher order powers of $p$ are negligible for small values of $n, P$ will be approximated by $n p$ and will thus be an approximately linear function of $n$. For example, if $p=.01$ and $n=6$,

$$
P=6 p-15 p^{2}+20 p^{3}-15 p^{4}+6 p^{5}-p^{6}=.0585199
$$

(Manuscript received April 24, 1987; revision accepted for publication December 11, 1987.) 